\section{Serial Nuclear Magnetic Resonance Imaging in Acute Myocardial Infarction}

\author{
LEE R. DILWORTH, MD \\ ALEX M. AISEN, MD \\ G.B. JOHN MANCINI, MD \\ ANDREW J. BUDA, MD
}

\begin{abstract}
$\mathbf{R}$
ecent studies show that proton nuclear magnetic resonance (NMR) imaging can detect myocardial ischemia and acute myocardial infarction (AMI) in animal models ${ }^{1-3}$ and in humans. ${ }^{4-6}$ The area of AMI appears as increased signal intensity on the spin-echo NMR images and most likely reflects the regional edema associated with tissue necrosis. ${ }^{1}$ Thus, the time course of regional edema and the evolution of infarct healing may be revealed by serial NMR studies. In a recent canine study, Pflugfelder et $\mathrm{al}^{7}$ examined the time course of the increased NMR signal intensity associated with AMI. They found that the relative signal intensity increased between the day of AMI and 2 weeks after AMI and subsequently decreased by the 20th day. We examined the early time course of NMR changes in humans
\end{abstract}

The 9 patients who were examined in our study consisted of 8 men and 1 woman, aged 38 to 71 years (mean 50). All patients presented with an initial Qwave AMI, which was anterior in 5 and inferior in 4. Seven patients underwent acute coronary reperfusion using thrombolytic agents or percutaneous transluminal coronary angioplasty at a mean interval of 4.5 hours after initial onset of chest pain. Patient imaging was performed with a 0.35 -tesla NMR system using a dual spin-echo pulse sequence electrocardiographicully gated to the $R$ wave. The first imaging study was done 3 to 5 days after AMI, after discharge from the coronary care unit. Each patient was restudied 10 to 14 days after AMI, just before hospital discharge. First $\left(S F_{1}\right)$ and second $\left(S E_{2}\right)$ spin-echo images were obtained using transaxial and coronal acquisition planes with the patient in the $30^{\circ}$ right anterior oblique position to obtain true long-axis projections through the heart. ${ }^{8}$ Signal intensities of infarcted $\left(I_{\text {infarct }}\right)$ and remote normal $\left(I_{\text {normal }}\right)$ myocardium were measured from operator-defined regions of interest (Fig. 1). Euch region of interest contained at least 100 voxels

From the Cardiology Division, Department of Internal Medicine, and the Department of Radiology, University of Michigan Medical School, 1500 East Medical Center Drive, Ann Arbor, Michigan 48109-0366. This work was supported in part by a grant-in-aid from the American Heart Association of Michigan Lathrup Village, Michigan; Grant HL29716 from the National Heart, Lung, and Blood Institute, National Institutes of Health, Bethesda, Marvland; and the Veterans Administration, Washington. D.C. Manuscript received August 1, 1986; revised manuscript received and accepted December 29, 1986. and generally were 100 to 150 voxels in size. Signal intensity values were expressed as normalized values using the following relation: $l_{\text {infurct }}-1_{\text {nornal }} / l_{\text {normal }} \times$ $100 \%$. The inter- and intraobserver variability for intensity measurements in the normal myocardium was $25.8 \%$ and $15.0 \%$, respectively, and in the infarcted myocardium, $12.4 \%$ and $9.1 \%$. $T_{2}$ magnetic relaxation times were calculated from infarct and normal regions according to the formula: $\left(T E_{2}-T E_{1}\right) / \log _{e}\left(I_{1}\right)$ $I_{2}$, where $T E_{2}=$ time to $S E_{2} ; T E_{1}=$ time to $S E_{1} ; I_{1}$ and $I_{2}=$ intensity of $S E_{1}$ and $S E_{2}$. Mean percent variability of $\mathrm{T}_{2}$ of fat and skeletal muscle were $9.2 \%$ and $24.2 \%$, respectively.

All data are expressed as mean \pm standard error of the mean. Statistical analysis was performed using the Student $\mathrm{t}$ test. A $p$ value was considered significant at the $<0.05$ level.

In each patient, an area of increased signal intensity was noted on the initial NMR study in a location corresponding to the electrocardiographic location of the Q-wave infarct. There was some variability in the ease with which the infarct location was identified on visual inspection of the $S E_{1}$ and $S E_{2} N M R$ images (Fig. 2). The infarct location was sometimes not apparent on certain tomographic projections and was more apparent with others. The electrocardiogram was used to help localize the NMR abnormality for further quantitative analysis

The intensity values for normal and infarcted regions are shown in Figure 3. For both the $S E_{1}$ and $S E_{2}$ images the intensity of the infarcted myocardium was significantly greater than that for the remote normal myocardium. 'I'his was true both for the early postinfarction studies and the late predischarge studies. When the regional intensity values were nurmulized, $S E_{1}$ was $37 \pm 13 \%$ on initial study and increased to 51 $\pm 17 \%$ on the predischarge study, but this change did not achieve statistical significance. The $S E$, normalized regional intensity value tended to decrease from acute to predischarge studies, from $89 \pm 33 \%$ to $53 \pm$

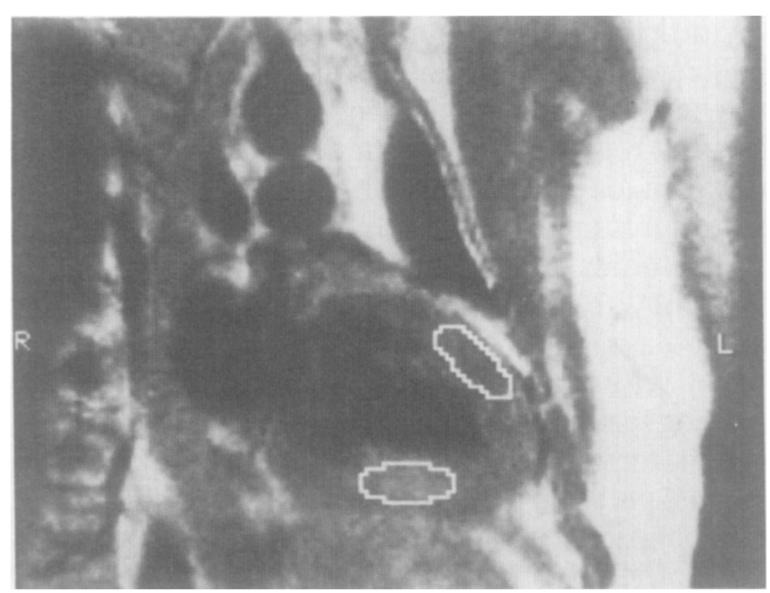

FIGURE 1. Regions of interest are outlined in normal (upper) and infarcted (lower) regions of the left ventricle in a patient with an acute inferior myocardial infarction. Note the increased signal intensity in the inferior (lower) zone. 


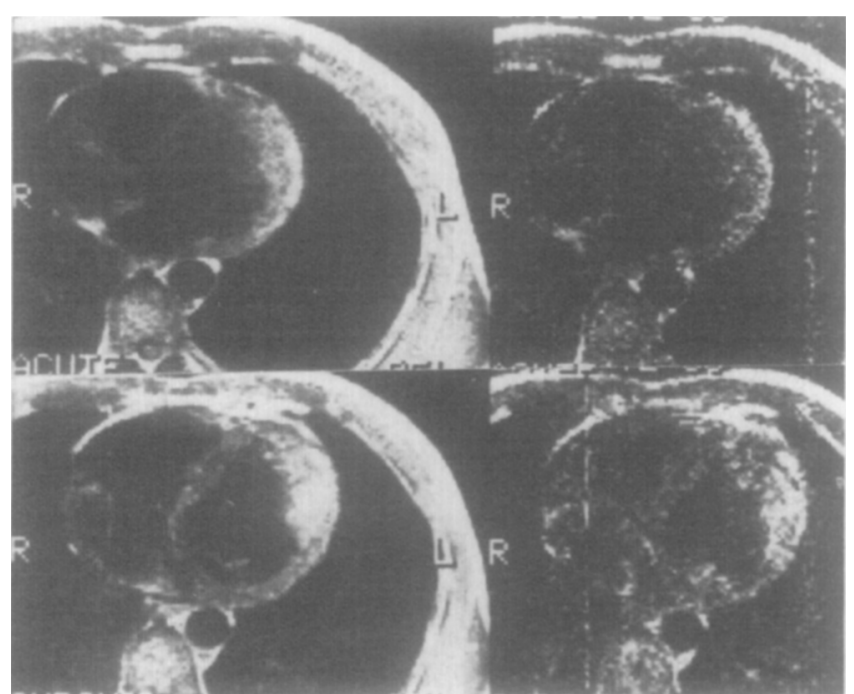

FIGURE 2. First ( $\mathrm{SE}_{1}$, left) and second $\left(\mathrm{SE}_{2}\right.$, right) spin-echo nuclear magnetic resonance images in a patient with anterolateral myocardial infarction. Top, acute studies; bottom, predischarge (chronic) studies. The area of infarction appears as an area of increased signal intensity and is easily appreciated on both $\mathrm{SE}_{1}$ and $\mathrm{SE}_{2}$ images. There is little change in the signal intensities between the acute and predischarge studies.

$17 \%$ ( $p>0.05$ ), but again this change did not reach statistical significance.

The acute $\mathrm{T}_{2}$ magnetic relaxation time measured 34 $\pm 3 \mathrm{~ms}$ in the remote normal myocardium and was significantly increased in the infarct region $[48 \pm 4 \mathrm{~ms}$, $p<0.05$ ). On the predischarge study, the $T_{2}$ relaxation time was prolonged in the remote normal myocardium (51 $\pm 5 \mathrm{~ms}, p<0.05)$. The predischarge $T_{2}$ values in the infarct region also were prolonged $(72 \pm 27 \mathrm{~ms})$. When the $T_{2}$ relaxation times were expressed as normalized values, the normalized $T_{2}$ relaxation time was $51 \pm 22 \%$ during initial study and remained elevated at $33 \pm 39 \%$ on the predischarge NMR study. Although there was a trend to some improvement in the normalized $\mathrm{T}_{2}$ values, this did not reach statistical significance.

Until the present study, the time course of NMR abnormalities associated with AMI is man was unknown. Since these NMR changes may reflect alterations in regional edema and may indicate evolutionary changes in the healing process, further serial studies in humans are important. Our data suggest that serial studies during the early in-hospital phase of AMI show changes in signal intensity ratios and $T_{2}$ magnetic relaxation times in the infarct zone which persist, with no statistically significant interval change. This suggests that regional edema in the infarct zone persists for at least 2 weeks after initial infarction.

Most patients in our study underwent thrombolytic or mechanical reperfusion. Reperfusion may have enhanced the regional edema and subsequent NMR abnormality, and thus may have altered the subsequent time course of NMR change. Our data, however, do agree with those of Pflugfelder et al, 7 who studied dogs for several weeks after complete coronary occlusion without reperfusion. Although they noted a significant

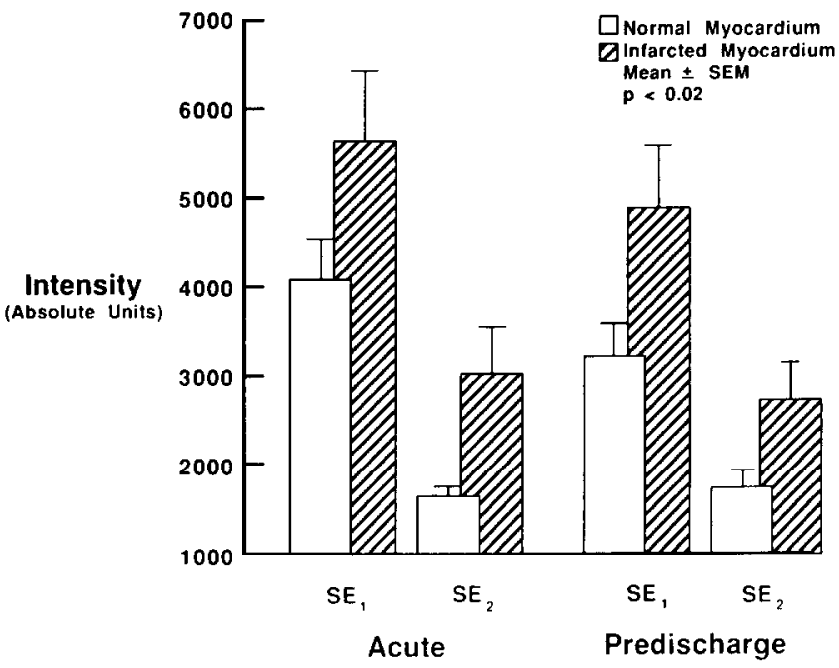

FIGURE 3. Absolute intensity values in normal and infarcted myocardial regions demonstrate the relative increased signal intensity of infarcted tissue on $\mathrm{SE}_{1}$ and $\mathrm{SE}_{2}$ images. The change in normalized infarct intensity from initial to predischarge study was not statistically significant.

increase in signal intensity between the initial day of AMI and day 14 after AMI, examination of their data does not indicate significant change in the NMR abnormality.

Our data further emphasize limitations of using magnetic relaxation times to characterize myocardial ischemia and injury in humans. $\mathrm{T}_{1}$ relaxation times were not calculated because additional pulse sequences would have been necessary, which would have led to unacceptable patient study times, almost 2 hours. In our experience, $\mathrm{T}_{1}$ times calculated from cardiac studies have been too variable to be clinically useful. The $\mathrm{T}_{2}$ times were calculated from signal intensity values as previously described. The fact that the $T_{2}$ values increased significantly in the remote nonischemic myocardium in our study population suggests that $T_{2}$ relaxation times as calculated in the present study may have only limited value. Our data suggest that $T_{2}$ times in patient studies need to be interpreted cautiously until the specific determinants of $\mathrm{T}_{2}$ are better understood and until improved methods for its calculation are developed.

Acknowledgment: We thank Sheree Wilson for excellent secretarial assistance.

1. Higgins CB, Herfkens R, Lipton MJ, Sievers R, Sheldon P, Kaufman L, Crooks EE. Nuclear magnetic resonance imaging of acute. Myocardial infarction in dogs: alterations in magnetic relaxation times. Am J Cardiol 1983; $52 ; 184-188$.

2. Pflugfelder PW, Wisenberg G, Prato FS, Carroll SE, Turner KL. Early detection of canine myocardial infraction by magnetic resonance imaging in vivo. Circulation 1985,71:587-594

3. Buda AJ, Aisen AM, Juni [E, Gallagher KP, Zotz RJ. Detection and sizing of myocardial ischemia and infarction by nuclear magnetic resonance imaging in the canine heart. Am Ileart I 1985:110:1284-1290.

4. Wesbey G, Higgins CB, Lanzer P, Botvinick E, Lipton M]. Imaging and characterization of acute myocardial infraction in viva by grated muclear magnetic resonance. Circulation 1984;69:125-130.

5. McNamara MT, Higgins CB, Schechtmann N, Botvinick E, Lipton M Chatteriee K. Amparo FG. Detertion and charasterization of acute myocardial infarction in man with use of gated magnetic resonance. Circulation $1985: 71: 717-724$

6. Johnston DI, Thompson RC, J,iı P, Dinsmore RE, Wismer GI, Saini S, Ka11 
S. Rosen BR, Brady TJ, Okada TD. Magnetic resonance imaging during acute myocardial infarctivin. An / Cardiol 1986;57:1059-1065.

7. Pflugfelder PW. Wisenberg G. Prato FS. Turner KL, Carroll SE. Serial imaging of canine myocardial infarction by in vivo nuclear magnetic reso- nance. $/ A C C, 1986 ; 7: 84: 3-849$

8. Dinsmore RE, Wismer GL, Levine RA, Okalla RI). Brady 'I]. Mugnetic resonance imaging of the heart: positioning and gradient angle selection for optimal imaging planes. AJR 1984;143:1135-1142

\section{Angiographic Demonstration of Congenital Intercoronary Communication in Normal Adults}

\author{
GERARDO VOCI, MD \\ RAVINDRA B. PATEL, MD \\ ATUL D. TRIVEDI, MD \\ PIYUSH V. PATEL, MD \\ ALFRED C. BURRIS, MD \\ SAMUEL R. RUBY, MD
}

P rominent anastomuses between the right and left coronary arteries, which are present during fetal life, persist into the postnatal period and diminish in caliber by the 8 th month. ${ }^{1}$ Recent anatomic studies indicate that the normal adult heart contains a profusion of small interconnecting vessels less than $200 \mu$ in diameter. ${ }^{2}$ Their angiographic demonstration in living humans with normal coronary arteries is probably precluded by minimal or no flow across them and by limitation of resolution of imaging systems. We report angiographic documentation of a prominent intercoronary conmection between a dominant right and nondominant left circumflex coronary arteries in 3 normal adults.

During the last 10 years, 6,400 coronary arteriographic studies have been performed in our laboratory. Normal arteriograms recorded from 600 patients investigated for atypical chest pain, cardiomyopathy and valvular heart disease were examined and 3 subjects were identified who had the intercoronary connection described in this report.

Clinical features are summarized in Table I. The studies were carried out using the transfemoral technique with standard $7 F_{r}$ catheters. There was no pressure damping during selective placement of the catheter tip in either coronary ostium. Multiple oblique and hemiaxial views were obtained.

In all 3 cases injection into the normal right coronary artery resulted in prompt visualization of the left circumflex artery through a prominent straight connection, at or near the level of the crux, measuring after correction for magnification 1.5, 1.1 and $1.6 \mathrm{~mm}$ in caliber, respectively. In cases 1 and 2 the left circumflex artery was visualized in its entirety (Fig. $1 \mathrm{~A}$ and $2 A$ ). Additionally, in case 2 the retrograde filling progressed to partially visualize the left main and the

From the Cardiology Section, Department of Medicine, Episcopal Hospital and Temple University Medical School, Philadelphia, Pennsylvania 19125, and the Medical Center Hospital. Midland, Texas 79701. Manuscript received September 8, 1986; revised manuscript received and accepted December 22, 1986. left anterior descending arteries. In case 3 retrograde filling of the distal left circumflex artery only was observed (Fig. 3A).

During injection of the left coronary artery a minimal amount of contrast medium crossed the connection to partially fill the right coronary artery in case 1 (Fig. $1 B$ ) and not in cases 2 and 3 . To account for this apparent discrepancy one may propose that both the force of injection and the velocity of flow in the left coronary artery would suffer some degree of dispersion because of its larger vascular capacity. A selective injection into the left circumflex artery probably would have resulted in full visualization of the right coronary artery. Angiographic luminal integrity of the left coronary arterial system is evident in all 3 cases. (Fig. $1 B$ to $3 B$ ).

The prominent straight connection between 2 major nonobstructed right and left circumflex coronary arteries identified in each patient, at or near the level of the crux, is incontrovertible. It is clearly different from the inter- and intracoronary anastomoses seen in the course of occlusive coronary artery disease. Acknowledged for many years in most patients with obstructive coronary artery disease, these anastomotic channels consist of 1 or more small, tortuous channels of differing sizes and lengths, carrying a variable amount of flow and functioning only upon the demand of a severe perfusion gradient. ${ }^{3}$ Only on rare occasions have they been found to be of somewhat larger caliber.

Presence of large anastomotic connections in the absence of obstructive coronary artery disease, as seen in these 3 cases, is extremely rare. A careful literature search revealed only 3 previously reported cases ${ }^{4,5}$ with intercoronary connection strikingly similar in all details to that reported here. In 1 of the 2 cases described by Hines et al, ${ }^{4}$ a 67 -year-old man, extensive obstructive coronary pathology was also present. In

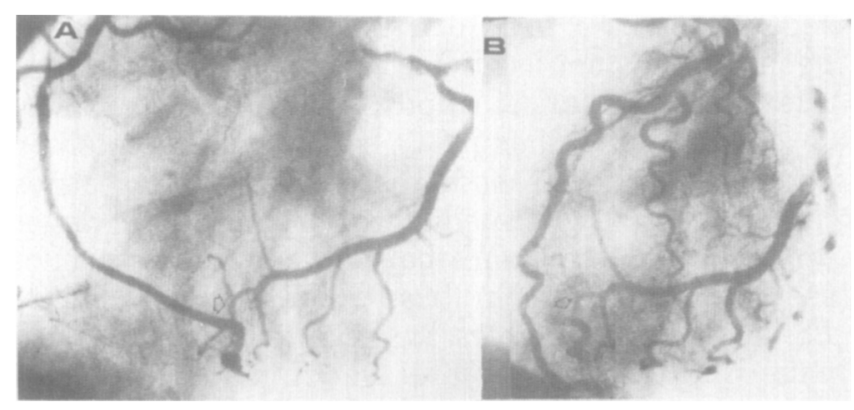

FIGURE 1. Case 1. A, selective injection of the right coronary artery, left anterior oblique view, demonstrating retrograde filling of entire left circumflex artery via a 1.5-mm-diameter connection at level of crux (arrow). $B$, selective injection of the left coronary artery, left anterior oblique view, demonstrating visualization of distal right coronary artery via the existing connection (arrow). 